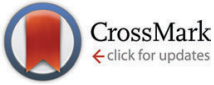

Cite this: Chem. Commun., 2016, 52,8846

Received 29th January 2016, Accepted 22nd June 2016

DOI: $10.1039 / c 6 c c 05147 b$

www.rsc.org/chemcomm

\section{Direct amidation of unprotected amino acids using $\mathrm{B}\left(\mathrm{OCH}_{2} \mathrm{CF}_{3}\right)_{3}^{\dagger}$}

\author{
Rachel M. Lanigan, $\ddagger^{a}$ Valerija Karaluka, $\ddagger^{a}$ Marco T. Sabatini, $\ddagger^{a}$ Pavel Starkov, $\S^{a}$ \\ Matthew Badland, ${ }^{b}$ Lee Boulton ${ }^{\mathrm{c}}$ and Tom D. Sheppard*a
}

\begin{abstract}
A commercially available borate ester, $\mathrm{B}\left(\mathrm{OCH}_{2} \mathrm{CF}_{3}\right)_{3}$, can be used to achieve protecting-group free direct amidation of $\alpha$-amino acids with a range of amines in cyclopentyl methyl ether. The method can be applied to the synthesis of medicinally relevant compounds, and can be scaled up to obtain gram quantities of products.
\end{abstract}

Amino acids are valuable renewable chemical building blocks for the synthesis of a wide range of natural products, pharmaceuticals and agrochemicals, and can serve as a source of chirality for a range of ligands and catalysts. However, their use in synthesis relies heavily on protecting group chemistry, ${ }^{1}$ with either the amine or the carboxylic acid requiring protection before key bond-forming processes are carried out. Amidation is one of the most common transformations used in the synthesis of many industrially important molecules, ${ }^{2}$ and amino acid amides form part of the core of numerous globally important pharmaceuticals including sitagliptin, ramipril and lacosamide (Scheme 1). ${ }^{3}$ The synthesis of amino acid amides typically proceeds via a three step sequence involving protection of the nitrogen atom with a carbamate (Boc, Fmoc, Cbz) before amidation of the carboxylic acid and subsequent deprotection. Nitrogen protection is usually essential as self-reaction of the free amino acid can lead to the formation of diketopiperazines, oligomers or polymers. The use of carbamate protecting groups is highly inefficient as they have a relatively high molecular weight in comparison to the amino acid itself (Boc 101; Fmoc 223; Cbz 135), and their introduction and

\footnotetext{
${ }^{a}$ Department of Chemistry, University College London, Christopher Ingold Laboratories, 20 Gordon St, London, WC1H OAJ, UK.

E-mail: tom.sheppard@ucl.ac.uk

${ }^{b}$ Pfizer Global Pharmaceutical Sciences, Discovery Park, Ramsgate Road, Sandwich, Kent, CT13 9NJ, UK

${ }^{c}$ GlaxoSmithKline, Medicines Research Centre, Gunnels Wood Road, Stevenage, Herts, SG1 2NY, UK

$\dagger$ Electronic supplementary information (ESI) available: Full experimental procedures and ${ }^{1} \mathrm{H}$ and ${ }^{13} \mathrm{C}$ data for all compounds. See DOI: $10.1039 / \mathrm{c} 6 \mathrm{cc} 05147 \mathrm{~b}$ \$ These authors contributed equally.

$\S$ Current address: Department of Chemistry, Tallinn University of Technology, 15 Akadeemia Rd, 12618 Tallinn, Estonia.
}

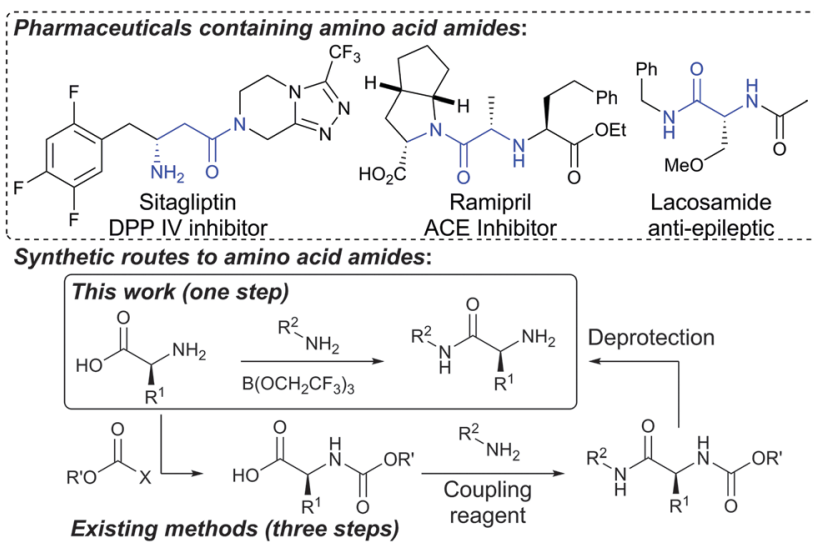

Scheme 1 Important amino acid amides and the typical synthetic route used to prepare them.

removal requires additional reagents, solvents and purification materials. A more straightforward approach would be the direct amidation of the free amino acid to give the amino amide in a single step. This has rarely been achieved, however, with only scattered reports in the literature that have not been widely applied. ${ }^{4}$ The only reasonably effective method involves reaction of the amino acid with the highly toxic gas hexafluoroacetone, followed by subsequent reaction with an amine. ${ }^{5}$ Although great progress has been made in recent years on the development of new amidation methods ${ }^{6}$ which make use of low-cost reagents ${ }^{7,8}$ or catalysts ${ }^{9}$ that are easy to separate from the amide products, few of these methods are applicable to highly functionalized compounds and none have been applied to the direct amidation of free amino acids. ${ }^{10}$

We have recently reported the use of borate esters, and $\mathrm{B}\left(\mathrm{OCH}_{2} \mathrm{CF}_{3}\right)_{3}{ }^{11}$ in particular, for the direct amidation of a wide range of acids and amines, including functionalized examples. ${ }^{8}$ An important advantage of this method is the ease with which the amide products can be purified using a simple filtration work-up involving commercially available resins, which scavenge unreacted acid and amine as well as any boron-containing byproducts. 


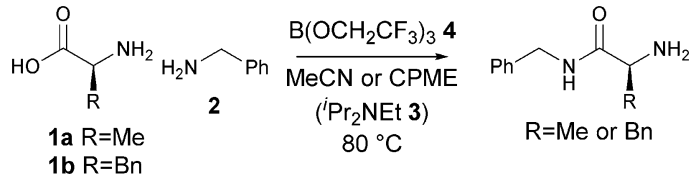

Scheme 2 Direct amidation of alanine or phenylalanine using benzylamine.

Table 1 Direct amidation of $\mathbf{1 a}$ or $\mathbf{1 b}$ and $\mathbf{2}$ under a variety of conditions

\begin{tabular}{lllllll}
\hline Entry & $\mathrm{R}$ & Solvent & 2 (eq.) & 3 (eq.) & 4 (eq.) & Yield $^{a}$ \\
\hline 1 & Me & MeCN & 1 & 1 & 1 & $(35)$ \\
2 & Me & MeCN & 1 & 1 & 3 & $(71)$ \\
3 & Me & MeCN & 1 & 1 & 0 & $(0)$ \\
4 & Bn & MeCN & 1 & 1 & 3 & 61 \\
5 & Bn & CPME & 1 & 1 & 3 & 65 \\
6 & Bn & CPME & 3 & 0 & 3 & 95 \\
7 & Bn & CPME & 3 & 0 & $3^{b}$ & $(9)$ \\
8 & Bn & CPME & 3 & 0 & 0 & $(0)$
\end{tabular}

${ }^{a}$ Yields measured by ${ }^{1} \mathrm{H}$ NMR using naphthalene as an internal standard; conversions are shown in parentheses. ${ }^{b} \mathrm{~B}(\mathrm{OMe})_{3}$ was used instead of 4 .

In this paper, we demonstrate that $\mathrm{B}\left(\mathrm{OCH}_{2} \mathrm{CF}_{3}\right)_{3}$ is a highly effective reagent for the direct amidation of unprotected amino acids to give useful $\alpha$-amino amide products in a single step. This has the potential to significantly shorten the synthesis of many pharmaceutically relevant compounds.

We began our investigation (Scheme 2 and Table 1) by exploring the direct amidation of alanine 1a with benzylamine 2 (entry 1). Under our previous amidation conditions (MeCN, reflux), ${ }^{8}$ addition of a base (diisopropylethylamine 3 ) was necessary to solubilise alanine in the reaction. We observed a promising $35 \%$ conversion to the desired amide using one equivalent of $\mathrm{B}\left(\mathrm{OCH}_{2} \mathrm{CF}_{3}\right)_{3} 4$, with little evidence for significant self-reaction of the amino acid. Addition of excess borate 4 increased the conversion to $71 \%$ (entry 2). Crucially, in the absence of the borate reagent no conversion to the amino amide was observed (entry 3). As the amidation product derived from alanine was extremely polar and time-consuming to isolate, we switched our attention to the direct amidation of phenylalanine $\mathbf{1 b}$ with benzylamine $\mathbf{2}$. Under similar conditions, this amide was obtained in $61 \%$ yield (entry 4). A screen of different solvents, ${ }^{12}$ led us to identify cyclopentyl methyl ether (CPME) ${ }^{13}$ as a promising solvent for the amidation reaction (entry 5). A significant improvement in the yield was seen by increasing the number of equivalents of benzylamine 2 used (entry 6), and with excess benzylamine an additional base was no longer required. The importance of using $\mathrm{B}\left(\mathrm{OCH}_{2} \mathrm{CF}_{3}\right)_{3}$ as the coupling reagent was underlined by the fact that $\mathrm{B}(\mathrm{OMe})_{3}{ }^{8 a}$ led to only $9 \%$ conversion under otherwise identical reaction conditions (entry 7). As observed previously with alanine, no product was formed in the absence of the borate reagent 4 (entry 8).

With an effective procedure in hand, we then went on to examine the scope with regard to the amino acid component (Scheme 3). In order to facilitate purification of the products we used $n$-propylamine as the amine so the products could be purified via a simple solid-phase work-up and subsequent

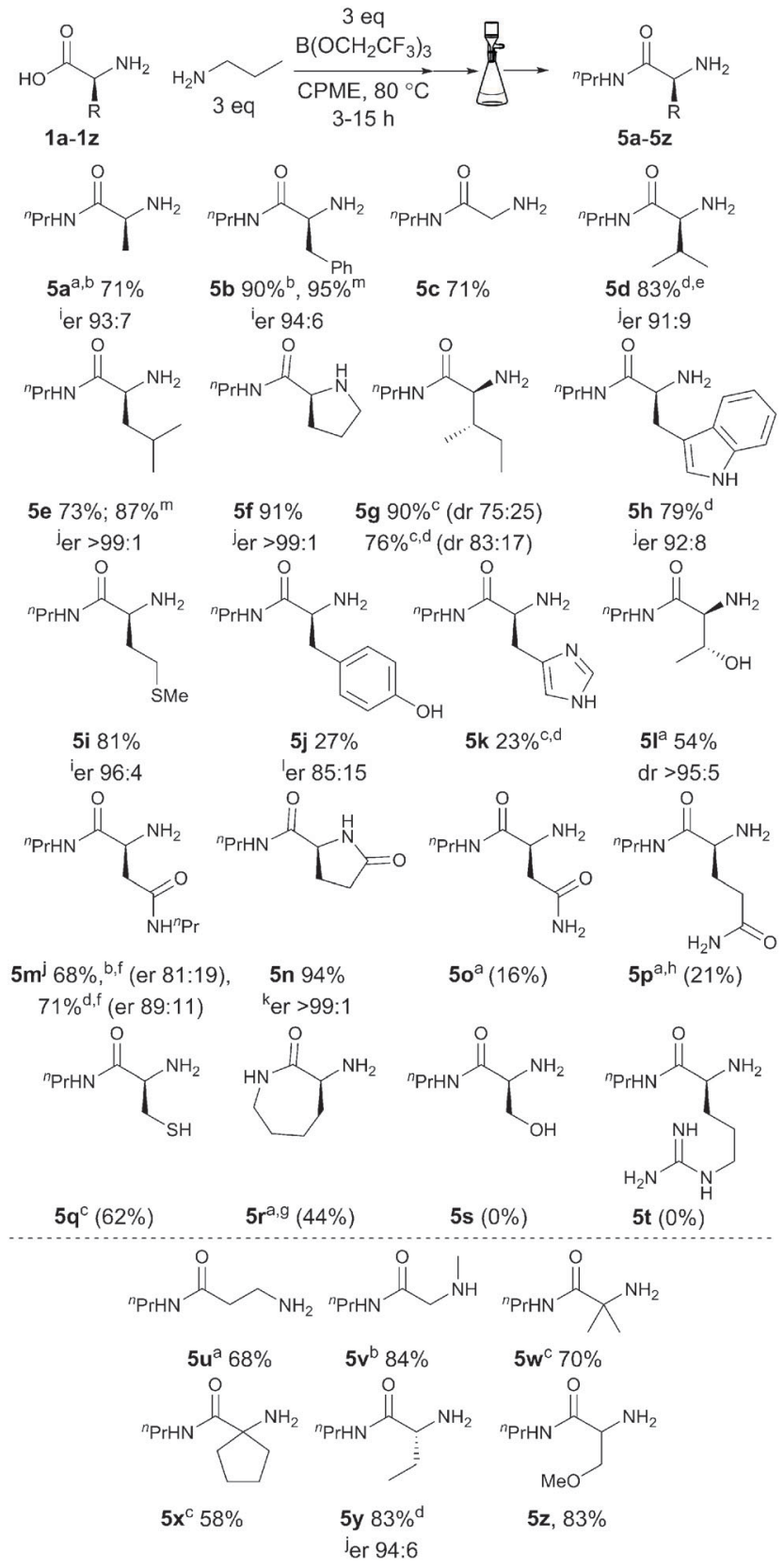

Scheme 3 Direct amidation of unprotected amino acids with propylamine; isolated yields (NMR yields shown in parentheses); ${ }^{14}$ a reaction carried out in $\mathrm{MeCN}$; ${ }^{b}$ isolated as $\mathrm{HCl}$ salt; ${ }^{c} 125{ }^{\circ} \mathrm{C}$; ${ }^{d}$ borate 4 was added dropwise over $1 \mathrm{~h} ;{ }^{e} 110{ }^{\circ} \mathrm{C} ;{ }^{f} 4$ eq. of 4 and 6 eq. propylamine; ${ }^{g}$ no propylamine; ${ }^{h} 15 \%$ yield of $\mathbf{5 n}$ also observed; ${ }^{i}$ determined using imine formation with a chiral aldehyde, ${ }^{15}{ }^{j}$ determined using Marfey's reagent; ${ }^{16}$ ${ }^{k}$ determined using a chiral shift reagent, ${ }^{17}$ ' determined by HPLC of a derivative using a chiral stationary phase; ${ }^{m} 1 \mathrm{~g}$ scale.

evaporation of the volatile materials. The direct amidation products $\mathbf{5 a}-\mathbf{5 b}$ derived from alanine and phenylalanine were obtained in $71 \%$ and $90 \%$ isolated yield respectively. Other amino acids containing alkyl/aryl side chains including glycine, valine, leucine, proline and isoleucine also underwent direct amidation in high yield $(\mathbf{5 c - 5 g})$, although in the case of isoleucine a significant amount of epimerization was observed, which 
could be reduced by reduction of the reaction time and by adding the borate reagent dropwise.

Pleasingly, tryptophan (5h) and methionine (5i) were also good substrates for the direct amidation reaction, giving high yields of the corresponding amides. Even amino acids with functionalized side chains were compatible with the reaction conditions, with the amides derived from tyrosine (5j) and histidine (5k) being obtained in moderate yield, and threonine undergoing direct amidation in an impressive yield of 54\%, despite the presence of the free hydroxyl group (5l). Aspartic acid underwent double amidation in the presence of excess amine/borate to give the diamide $5 \mathbf{m},{ }^{18}$ whereas in the case of glutamic acid, lactam formation took place alongside amidation to give 5n. A small improvement in both the yield and enantiopurity was seen by adding the borate dropwise to the reaction mixture. Asparagine and glutamine were poor substrates for the reaction giving only low conversions to the desired amides 5o-5p. Whilst reaction of cysteine led to a good conversion to the desired amide $\mathbf{5 q}$, we were unable to purify this amide despite numerous attempts. Lactamisation of lysine outcompeted the desired amidation reaction with $\mathbf{5 r}$ being the only product observed either in the presence or absence of propylamine. Direct amidation of serine and arginine did not give any of the expected amino amide. The reaction of serine led to a complex mixture of products with no observable amide 5s, a surprising result given the successful amidation of threonine. Arginine was insoluble in the reaction mixture and no 5t was observed. Non-proteinogenic amino acids also underwent direct amidation under these conditions (5u-5z) with $\beta$-alanine, sarcosine and $\alpha$-aminoisobutyric acid giving the corresponding amides $\mathbf{5 u - 5 w}$ in good yield. Relatively hindered cyclopentyl amino acid amide $\mathbf{5 x}$ was obtained in moderate yield, whereas 2 -aminobutyric acid and $O$-methylserine gave the corresponding amides $\mathbf{5 y}-\mathbf{5 z}$ in excellent yield. Pleasingly, the vast majority of the amides $\mathbf{5 a}-\mathbf{5 z}$ were obtained in high purity from a simple filtration without the need for chromatography or an aqueous work-up. Furthermore, scale-up of two amidation reactions $(\mathbf{5 b}, \mathbf{5 e})$ to $1 \mathrm{~g}$ scale resulted in improved yield of products whilst maintaining the same level of enantiopurity, demonstrating the synthetic utility of this procedure. Whilst a small degree of racemisation was observed with some amino acids, it should be noted that the amino amides (or simple derivatives such as their $\mathrm{HCl}$ salts) are usually crystalline so recrystallization could potentially be used to obtain enantiopure material if needed.

The scope of the reaction with regard to the amine component was investigated next (Scheme 4), through the preparation of amides derived from a selection of different amines. Simple aliphatic amines (6a-6f) including an unsaturated example (6c) worked well in the reaction. Amides could also be prepared from cyclic secondary amines in good yield (6g-6j). Importantly, it was also possible to prepare two dipeptide derivatives using amino acid tert-butyl esters as the nucleophile (6k-6l). The methodology was then applied to the synthesis of the previously reported anti-convulsant amino amides $6 \mathbf{m}-\mathbf{6} \mathbf{n}^{19}$ to demonstrate the application of the methodology. Finally, a synthesis of the anti-epileptic lacosamide 7 was carried out by

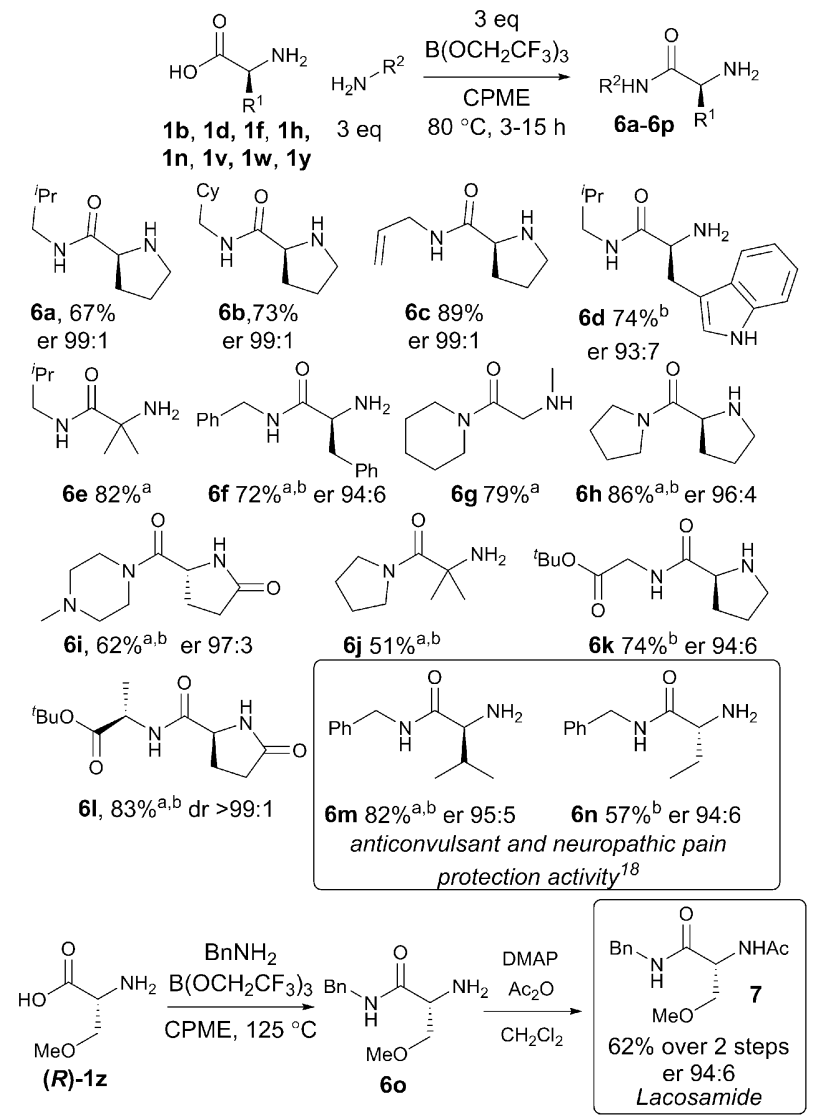

Scheme 4 Direct amidation reactions of amino acids with various amines; ${ }^{a} 125{ }^{\circ} \mathrm{C}$; ${ }^{b}$ borate 4 was added dropwise over $1 \mathrm{~h}$.

$$
\overbrace{\mathrm{R}^{1}}^{\mathrm{B}(\mathrm{OR})_{3}} \underset{\mathrm{R}=\mathrm{CF}_{3} \mathrm{CH}_{2}}{\mathrm{NH}_{2}}
$$

Scheme 5 Possible mechanism for the direct amidation of unprotected amino acids.

direct amidation of $(\boldsymbol{R})-\mathbf{1 z}$ with benzylamine, followed by acylation with acetic anhydride. ${ }^{20}$

A plausible mechanism for the amidation reactions is shown in Scheme 5. Coordination of both the amine and the carboxylic acid to the borate reagent should lead to cyclic intermediate 8 . $^{4, b}$ This can then undergo reaction with the amine partner to form the boron-bound amino amide 9. In cases where the amino acid and/or the amine are less reactive, racemisation could occur via borate-assisted deprotonation of intermediate 8 by the amine, leading to the formation of the formally aromatic heterocyclic enolate $\mathbf{1 0}$.

In summary, we have developed a practical and general method for the protecting-group free direct amidation of free amino acids. The amidation reaction is successful with 14 of 
the 20 common proteinogenic amino acids as well as six unnatural amino acids, and a variety of amines can be employed. In the majority of cases, the amino acid amide products can be isolated using a simple filtration work-up without the need for chromatographic purification. The utility of the method has been demonstrated by the synthesis of some medicinally relevant compounds.

We would like to thank the Engineering and Physical Sciences Research Council (EPSRC Advanced Research Fellowship to TDS; studentship to PS; Grant reference EP/E052789/1), the Department of Chemistry, University College London (studentship funding to RML and MS), Pfizer (EPSRC CASE award to VK) and GlaxoSmithKline (studentship support to MS). We would also like to acknowledge the EPSRC National Mass Spectrometry Facility at Swansea University for providing analytical services.

\section{Notes and references}

1 (a) I. S. Young and P. S. Baran, Nat. Chem., 2009, 1, 193-205; (b) T. Newhouse, P. S. Baran and R. W. Hoffmann, Chem. Soc. Rev., 2009, 39, 3010-3021.

2 (a) J. S. Carey, D. Laffan, C. Thomson and M. T. Williams, Org. Biomol. Chem., 2006, 4, 2337-2347; (b) L. Amarnath, I. Andrews, R. Bandichhor, A. Bhattacharya, P. J. Dunn, J. Hayler, W. Hinkley, N. Holub, D. Hughes, L. Humphreys, B. Kaptein, H. Krishnen, K. Lorenz, S. Mathew, G. Nagaraju, T. Rammeloo, P. Richardson, L. Wang, A. Wells and T. White, Org. Process Res. Dev., 2012, 16, 535-544; (c) D. J. C. Constable, P. J. Dunn, J. D. Hayler, G. R. Humphrey, J. L. Leazer Jr., R. J. Linderman, K. Lorenz, J. Manley, B. A. Pearlman, A. Wells, A. Zaks and T. Y. Zhang, Green Chem., 2007, 9, 411-420.

3 (a) G. A. Herman, C. Stevens, K. Van Dyck, A. Bergman, B. Yi, M. De Smet, K. Snyder, D. Hilliard, M. Tanen, W. Tanaka, A. Q. Wang, W. Zeng, D. Musson, G. Winchell, M. J. Davies, S. Ramael, K. M. Gottesdiener and J. A. Wagner, Clin. Pharmacol. Ther., 2005, 78, 675-688; (b) L. Pilote, M. Abrahamowicz, M. Eisenberg, K. Humphries, H. Behlouli and J. V. Tu, Can. Med. Assoc. J., 2008, 178, 1303-1311; (c) A. C. Errington, T. Stöhr, C. Heers and G. Lees, Mol. Pharmacol., 2008, 73, 157-169.

4 (a) S. H. van Leeuwen, P. J. L. M. Quaedflieg, Q. B. Broxterman, Y. Milhajlovica and R. M. J. Liskamp, Tetrahedron Lett., 2005, 46, 653-656; (b) S. H. van Leeuwen, P. J. L. M. Quaedflieg, Q. B. Broxterman and R. M. J. Liskamp, Tetrahedron Lett., 2002, 43, 9203-9207; (c) J. Li, K. Subramaniam, D. Smith, J. X. Qiao, J. J. Li, J. Qian-Cutrone, J. F. Kadow, G. D. Vite and B.-C. Chen, Org. Lett., 2012, 14, 214-217; (d) R. Sharma and R. Jain, Synlett, 2007, 603-606; (e) M. A. Schmidt, E. A. Reiff, X. Qian, C. Hang, V. C. Truc, K. J. Natalie, C. Wang, J. Albrecht, A. G. Lee, E. T. Lo, Z. Guo, A. Goswami, S. Goldberg, J. Pesti and L. T. Rossano, Org. Process Res. Dev., 2015, 19, 1317-1322.

5 J. Spengler, C. Böttcher, F. Albericio and K. Burger, Chem. Rev., 2006, 106, 4728-4746.

6 (a) R. M. Lanigan and T. D. Sheppard, Eur. J. Org. Chem., 2013, 7453-7465; (b) H. Lundberg, F. Tinnis, N. Selander and H. Adolfsson, Chem. Soc. Rev., 2014, 43, 2714-2742; (c) C. L. Allen and J. M. J. Williams, Chem. Soc. Rev., 2011, 40, 3405-3415; (d) R. García-Álvarez, P. Crochet and V. Cadierno, Green Chem., 2013, 15, 46-66; (e) V. R. Pattabiraman and J. W. Bode, Nature, 2011, 480, 471-479.
7 (a) J. R. Dunetz, Y. Xiang, A. Baldwin and J. Ringling, Org. Lett., 2011, 13, 5048-5051; (b) J. D. Goodreid, P. A. Duspara, C. Bosch and R. A. Batey, J. Org. Chem., 2014, 79, 943-954; (c) J. Bai, B. K. Zembrón and P. Vogel, Org. Lett., 2014, 16, 604-607.

8 (a) P. Starkov and T. D. Sheppard, Org. Biomol. Chem., 2011, 9, 1320-1323; (b) R. M. Lanigan, P. Starkov and T. D. Sheppard, J. Org. Chem., 2013, 78, 4512-4523; (c) V. Karaluka, R. M. Lanigan, P. M. Murray, M. Badland and T. D. Sheppard, Org. Biomol. Chem., 2015, 13, 10888-10894.

9 (a) S. Fatami, N. Gernigon and D. G. Hall, Green Chem., 2015, 17, 4016-4028; (b) N. Gernigon, R. M. Al-Zoubi and D. G. Hall, J. Org. Chem., 2012, 77, 8386-8400; (c) R. M. Al-Zoubi, O. Marion and D. G. Hall, Angew. Chem., Int. Ed., 2008, 47, 2876-2879; (d) H. Lundberg, F. Tinnis and H. Adolfsson, Chem. - Eur. J., 2012, 18, 3822-3826; (e) H. Lundberg and H. Adolfsson, ACS Catal., 2015, 5, 3271-3277; $(f)$ H. Lundberg, F. Tinnis and H. Adolfsson, Synlett, 2012, 2201-2204; $(g)$ K. Arnold, B. Davies, R. L. Giles, C. Grosjean, G. E. Smith and A. Whiting, Adv. Synth. Catal., 2006, 348, 813-820; (h) K. Ishihara, S. Ohara and H. Yamamoto, J. Org. Chem., 1996, 61, 4196-4197; ( $i$ ) C. L. Allen, A. R. Chhatwal and J. M. J. Williams, Chem. Commun., 2012, 48, 666-668; $(j)$ K. Arnold, A. S. Batsanov, B. Davies and A. Whiting, Green Chem., 2008, 10, 124-134; $(k)$ T. M. El Dine, W. Erb, Y. Berhault, J. Rouden and J. Blanchet, J. Org. Chem., 2015, 80, 4532-4544; (l) H. Morimoto, R. Fujiwara, Y. Shimizu, K. Morisaki and T. Ohshima, Org. Lett., 2014, 16, 2018-2021; $(m)$ N. Caldwell, C. Jamieson, I. Simpson and A. J. B. Watson, Chem. Commun., 2015, 51, 9495-9498; (n) S. Liu, Y. Yang, X. Liu, F. K. Ferdousi, A. S. Batsanov and A. Whiting, Eur. J. Org. Chem., 2013, 5692-5700; (o) T. M. El Dine, J. Rouden and J. Blanchet, Chem. Commun., 2015, 51, 16084-16087.

10 The use of a boronic acid catalyst for self-condensation of proline to the corresponding diketopiperazine has been reported, see ref. $8 h$; Amidation of some protected amino acid derivatives has also been reported, but the scope is limited in most cases: see ref. 8 , $9 a, k, n$ and $o$.

11 T. D. Sheppard, Tris(2,2,2-trifluoroethoxy)boron, ed. P. L. Fuchs, e-EROS Encyclopedia of Reagents for Organic Synthesis, John Wiley \& Sons, Ltd, 2014.

12 See ESI, $\dagger$ for further details.

13 K. Watanabe, N. Yamagiwa and Y. Torisawa, Org. Process Res. Dev., 2007, 11, 251-258.

14 For an eloquent justification for why 'enantiomeric ratio' should be employed as a measurement of enantiopurity, see: R. J. Gawley, J. Org. Chem., 2006, 71, 2411-2416.

15 S. M. Gibson, R. M. Lanigan, L. Benhamou, A. E. Aliev and T. D. Sheppard, Org. Biomol. Chem., 2015, 13, 9050-9054.

16 (a) P. Marfey, Carlsberg Res. Commun., 1984, 49, 591-596; (b) R. Bhushan and H. Brückner, Amino Acids, 2004, 27, 231-247.

17 W. H. Pirkle, D. L. Sikkenga and M. S. Pavlin, J. Org. Chem., 1977, 42, 384-387.

18 The relatively high degree of racemisation in the amidation of aspartic acid is most likely down to the in situ formation of the cyclic anhydride derivative under the dehydrating conditions.

19 A. M. King, C. Salom, J. Dinsmore, E. Salomé-Grosjean, M. De Ryck, R. Kaminski, A. Valade and H. Kohn, J. Med. Chem., 2011, 54, $4815-4830$.

20 Lacosamide is well-known to be prone to racemisation during the amidation/acylation steps in the synthesis: $(a)$ D. Choi, J. P. Stables and H. Kohn, J. Med. Chem., 1996, 39, 1907-1916; (b) P. Morieux, J. P. Stables and H. Kohn, Bioorg. Med. Chem. Lett., 2008, 16, 8968-8975; (c) S. Stecko, J. Org. Chem., 2014, 79, 6342-6346. 